\title{
The role of location and motion information in the tracking and recovery of moving objects
}

\author{
DaVID E. FenCSIK \\ Brigham and Women's Hospital, Boston, Massachusetts \\ and Harvard Medical School, Boston, Massachusetts \\ SARAh B. KLIEger \\ Brigham and Women's Hospital, Boston, Massachusetts \\ AND \\ TODD S. HoROWITZ \\ Brigham and Women's Hospital, Boston, Massachusetts \\ and Harvard Medical School, Boston, Massachusetts
}

\begin{abstract}
Observers in a multiple object tracking task can track about four to five independently moving targets among several moving distractors, even if all of the stimuli disappear for a 300-msec gap. How observers reacquire targets following such a gap reveals what kind of information they can maintain for targets. Previous research has suggested that participants maintain minimal information about a set of moving objects - namely, just their present spatial locations. We report five new experiments that demonstrate retention of location information for at least four objects, and extrapolated motion information for around two objects.
\end{abstract}

Much of what we interact with in the world is in motion. Drivers navigating through traffic, parents watching their children on a playground, and air-traffic controllers tracking planes on a runway and in the air; all must constantly track a set of moving things in order to know their current location and anticipate their future motion. The visual system must have mechanisms to track objects of interest as they move around the environment. In this article, we ask what kinds of information about an object may be tracked by these mechanisms.

Previous research has shown that people can successfully track around 4-5 targets out of 8-10 total moving objects (Pylyshyn \& Storm, 1988). This can be demonstrated using the multiple object tracking (MOT) task, in which observers are asked to keep track of a subset of stimuli ("targets") as they move independently around a display. After several seconds of tracking, all of the stimuli stop, and the observer indicates which stimuli were targets. In most cases, the primary dependent variable is the number of targets correctly selected.

In a typical MOT task, the stimuli are identical, so observers can only distinguish targets from distractors by following the trajectory of each stimulus during the trial. Using identical stimuli in the MOT task may seem unrealistically impoverished. After all, parents have multiple cues to distinguish their children from others on a playground, including clothing, hair color, and the sounds of their voices. Removing such cues could, in principle, re- duce the effectiveness of tracking mechanisms. However, using stimuli that have no such distinguishing characteristics still allows us to ask fundamental questions about the types of information that may be known about a set of moving objects. The two types of information we will focus on in this article are location information ("Do you know where your child is?") and motion information ("Do you know which way your child is running?").

Research incorporating the MOT task has been used to support visual index theory (e.g., Pylyshyn, 1989, 2001). This theory proposes a psychological mechanism that includes a set of tags ("indexes") that can be associated with a visible object in the environment. Indexes are "sticky," meaning that each index retains its association with an object as that object moves or changes appearance. Visual indexes are supposed to operate relatively early in visual processing, and their role is to assign priority for further processing to certain parts of the visual field (Pylyshyn, 1989). They do not require that an object be identified or that any of its perceptual features be detected. According to the theory, target tracking in an MOT task is mediated by the linking of an index to each target object. Thus, performance in the task depends on the number of individual indexes available.

Under the visual index theory, little information is extracted from each tracked stimulus - indexes mark the current spatial position of each stimulus, and no further information is needed to differentiate targets from distrac-

D. E. Fencsik, david.fencsik@csueastbay.edu 
tors. Consistent with this, if the stimuli used in an MOT task vary in color or shape, observers are poor at detecting changes in the features of tracked stimuli (Scholl, Pylyshyn, \& Franconeri, 1999; but see Bahrami, 2003). Additionally, they fail to use such features to discriminate targets from distractors (Klieger, Horowitz, \& Wolfe, 2004).

However, this does not necessarily mean that no further information can be used to track objects. Other representations may be used to support tracking, instead of or in addition to visual indexes (e.g., Oksama \& Hyönä, 2004; Yantis, 1992). These representations may enable retention of more than just location information.

In this article, we demonstrate that information beyond just current spatial location is retained for at least some of the target objects in MOT, and that such information may be used to aid tracking of moving objects. We do so by exploiting a recent discovery in MOT experiments: Observers are able to track successfully even if all of the stimuli simultaneously disappear for a brief (300- to 900-msec) gap (see Figure 1; Alvarez, Horowitz, Arsenio, DiMase, \& Wolfe, 2005; Horowitz, Birnkrant, Fencsik, Tran, \& Wolfe, 2006; Keane \& Pylyshyn, 2006). By investigating the information retained during a gap, we can determine how the visual system tracks moving objects.

Maintaining information about a tracking task during the simultaneous disappearance of all of the objects appears to depend on mechanisms distinct from those that generally support tracking of visible objects. Scholl and Pylyshyn (1999) showed that the temporary occlusion of one or two stimuli during tracking had no effect on tracking performance. However, when an object spontaneously disappeared and reappeared without occlusion cues, tracking performance declined markedly; the visual system seems to assume that a spontaneously disappearing object no longer exists, and stops tracking it (Scholl \& Feigenson, 2004). In contrast, Horowitz et al. (2006) found that, when all objects disappeared simultaneously, occlusion cues had no effect on tracking performance, and in some cases were detrimental. This dissociation suggests that the mechanisms that support tracking during the disappearance of one or a few objects may be distinct from those that support tracking through the simultaneous disappearance of all objects.

Further evidence comes from Alvarez et al. (2005), who showed that tracking is still possible when observers must perform a difficult visual search task (e.g., Wolfe, 1998) during a gap, and then resume tracking. Alvarez et al. argued that the tracking task may be temporarily postponed while search stimuli are selected for further processing. Horowitz et al. (2006) further argued that such postponement must depend on a memory system that can store information pertinent to the tracking task while it is suspended. Any information stored by this memory system must be available during the tracking task, since it cannot be extracted from the stimuli after they disappear.

To make the distinction between disappearance types more concrete, consider the example of driving a car on a highway. Other cars can individually disappear through occlusion, and tracking mechanisms must be able to deal with this situation. However, spontaneous disappearance of any one car is impossible, so there is no reason to be prepared to handle it. Such disappearance occurs only when the driver looks down at the dashboard, the radio, or a passenger, at which point all of the cars disappear simultaneously. The driver's ability to resume tracking the traffic depends on her having some memory of the environment from before she looked away. On the basis of research

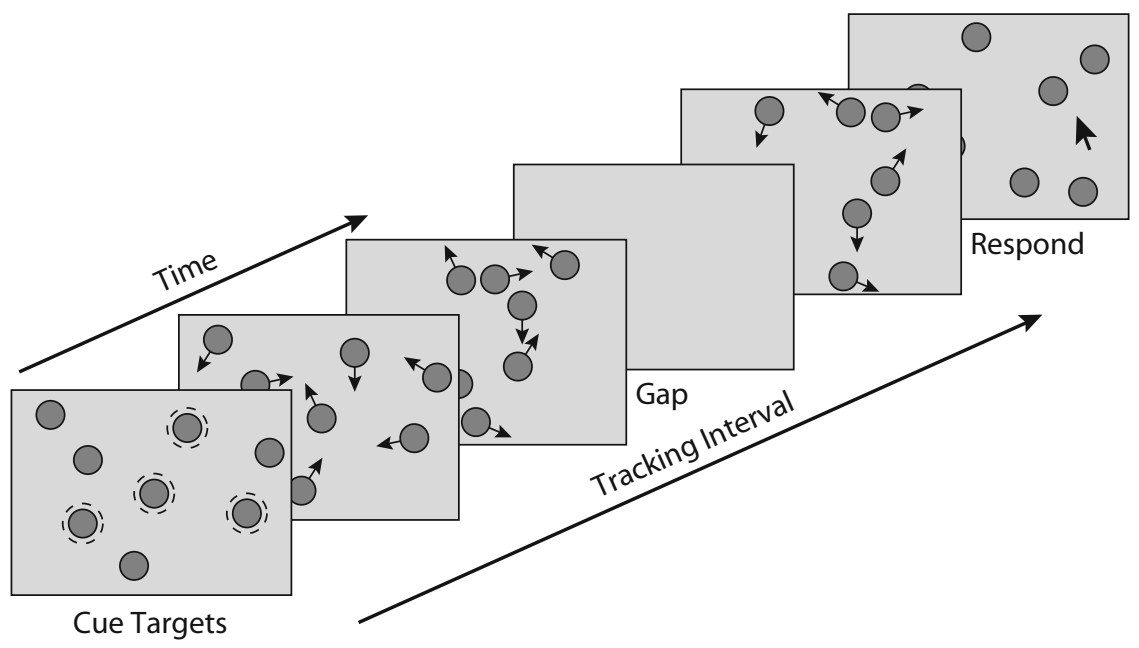

Figure 1. Illustration of multiple object tracking (MOT) through a gap. As in a typical MOT task, observers are presented with a set of identical stimuli, a subset of which are cued as targets. The cues disappear, and the observers must keep track of the targets during the tracking interval, while all of the stimuli move independently around the display. At some point during the tracking interval, all of the stimuli disappear simultaneously for a 300- to 900-msec gap, and then reappear simultaneously and continue moving. At the end of the tracking interval, all of the stimuli stop moving, and the observers indicate which stimuli are targets. 
summarized in the previous two paragraphs, we conclude that the driver's memory for traffic while her gaze is elsewhere depends on a mechanism distinct from the one that enables tracking while she is focused on the road.

By investigating what information may be stored by this memory system during a gap, we can elucidate how the visual system keeps track of moving objects. Depending on the information retained during the gap, different strategies can be used to reacquire targets following reappearance. On the one hand, if observers retain the pregap spatial locations of target stimuli, then they can search the area around those locations and identify the nearest stimuli as targets. On the other hand, if observers compute and store the velocities of any targets, then they can restrict their search along a target's path of motion and even anticipate where it will reappear. In this article, we will distinguish these two possibilities as storage of location information and storage of motion information, respectively.

Recently, Keane and Pylyshyn (2006) reported a series of experiments in which they investigated what kind of information may be used to reacquire targets following a gap in MOT. In one of their experiments (Experiment 3), all stimuli disappeared simultaneously for a 300- to 900msec gap, and then reappeared at one of three positions relative to their pregap positions (see Figure 2): In the "move" condition, stimuli reappeared as if they had continued moving at the same velocity while they were invisible; in the "no-move" condition, stimuli reappeared in the same positions they had occupied before the gap; in the "rewind" condition, stimuli reappeared at the positions they had occupied $x$ msec before the gap, where $x$ equals the gap duration. Thus, each stimulus "jumped" back along its trajectory by the same distance it would have moved forward in the move condition. In a block of trials, all stimuli reappeared under the same conditions, and then continued moving until the end of the trial.

In Keane and Pylyshyn's (2006) experiments, if observers maintained only the spatial locations of targets, then they should have been best at reacquiring them in the no-move condition and equivalent in the move and rewind conditions. If they maintained motion information, then tracking accuracy should have been highest in the move condition and progressively worse in the no-move and rewind conditions. In fact, observers' accuracy was equivalently high in both the no-move and rewind conditions, and substantially worse in the move condition. On the basis of these and other results, Keane and Pylyshyn concluded that observers retained the pregap locations of targets (along with some previous trajectory information), but no motion information.

However, Keane and Pylyshyn (2006) aside, there are reasons to believe that observers may retain some motion information. First, Scholl et al. (1999) found that observers could recall the direction of motion for targets in an explicit memory test. Second, observers can clearly encode more information from multiple stimuli than just spatial location (e.g., Kahneman, Treisman, \& Gibbs, 1992; Nissen, 1985; Vogel, Woodman, \& Luck, 2001), perhaps including motion information (Finke, Freyd, \& Shyi, 1986),

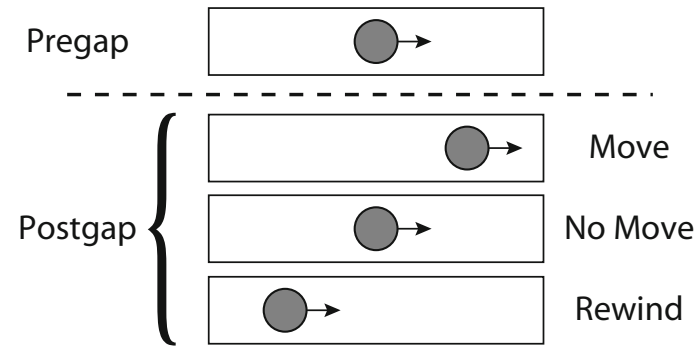

Figure 2. Variations in reappearance position following a gap in MOT. The top frame indicates a stimulus's position and velocity immediately before it disappears, and the other three frames indicate possible postgap reappearance positions relative to the pregap position. Note that the distance between the move and nomove positions is equal to the distance between the rewind and no-move positions. (See text for more details.)

so it is possible that some motion information is available to aid tracking. Finally, observers may have performed better in the no-move condition than in the move condition because using location information to reacquire targets is more efficient than using motion information, not because motion information is unavailable.

To further investigate the types of information that can be retained for target stimuli during a gap in MOT, we report two sets of experiments. In the first set, we extended the experiments described by Keane and Pylyshyn (2006). These experiments replicated their finding of superior tracking performance in the no-move condition relative to the move condition, but demonstrate in addition that observers do not remember a target's past trajectory any better than they anticipate its future motion path. In the second set of experiments, we compared performance in the move condition with a condition in which there was no movement information prior to the gap. The results of these experiments demonstrated that observers do use motion information to anticipate the reappearance positions of at least one or two tracked stimuli.

\section{EXPERIMENT 1}

Experiment 1 replicated and extended part of Experiment 3 from Keane and Pylyshyn (2006). Observers performed an MOT task in which all of the stimuli disappeared for a 300-msec gap. Following the gap, each stimulus reappeared either at the location it had occupied just prior to the gap (the no-move condition), at the location expected if it had continued moving at a constant velocity during the gap (the move condition), or at the position it had occupied $300 \mathrm{msec}$ before the gap (the rewind condition; see Figure 2). Each stimulus moved at a constant speed in a straight line, bouncing when it reached the edge of the display.

The purpose of this experiment was to test the predictions of two accounts of MOT performance with a gap. According to the location-matching account, observers retain only the pregap locations of the targets. On this account, target reacquisition efficiency, and hence overall tracking performance, should improve as the targets 
reappear closer to their pregap positions. Thus, accuracy should be equal in the move and rewind conditions and higher in the no-move condition. Alternatively, according to the motion-anticipation account, observers retain the pregap motion of each target. Using this information, they anticipate target reappearance positions, so accuracy should be highest in the move condition and increasingly worse in the no-move and rewind conditions.

In Experiment 1, reappearance condition was blocked. This was done so that observers could optimize their performance for each type of disappearance, if possible. However, we have conducted an unreported experiment with a mixed-block design and found no substantive difference in results. We report experiments with blocked designs here in order to replicate the experiments reported by Keane and Pylyshyn (2006).

\section{Method}

Participants. Twelve participants from the ages of 19 to 47 years ${ }^{1}$ ( $M=29.6$; 9 females) served as observers; 3 observers had participated in earlier tracking studies in our lab. Each had 20/25 corrected vision or better and passed the Ishihara color blindness test. All of the observers gave informed consent prior to the experiment and were paid $\$ 10 / \mathrm{h}$ for participating.

Apparatus. The observers were seated in a darkened room approximately $57 \mathrm{~cm}$ from the display. The stimuli were presented on a 21-in. monitor with a 75-Hz refresh rate (refresh duration of approximately $13.33 \mathrm{msec}$ ) controlled by a Macintosh G4 computer. The experimental procedure was generated in MATLAB 5.2.1, using the Psychophysics Toolbox extensions, version 2.53 (Brainard, 1997; Pelli, 1997)

Stimuli. The stimuli were dark gray disks with a black border drawn on a light gray background. Each disk subtended approximately $1.25^{\circ}$ of visual angle in diameter and had a border approximately $0.1^{\circ}$ thick. Each disk was assigned an initial direction and moved in that direction at a constant rate of approximately $8 \% \mathrm{sec}$, bouncing off the edge of the screen. The screen subtended approximately $40^{\circ} \times 30^{\circ}$. Movement trajectories were generated in advance and stored offline. All of the disks were at least $1.8^{\circ}$ apart (center to center), both on the first and last frames of the trial.

Procedure. At the beginning of each trial, the observers were presented with all 10 stimuli, randomly distributed on the display. The targets flashed four times over $4 \mathrm{sec}$, and then all of the stimuli moved along their trajectories for $5 \mathrm{sec}$ ( 376 frames). At a randomly selected point during the tracking interval, all of the stimuli disappeared simultaneously for $307 \mathrm{msec}$ ( 23 frames). This gap began at least $2 \mathrm{sec}$ after the tracking interval started and at least $1 \mathrm{sec}$ before it ended. After the tracking interval, all of the stimuli stopped, and the observers responded by selecting 4 stimuli with the mouse. During response, the observers received visual and auditory feedback indicating the accuracy of their selection. After they had selected 4 stimuli, they were informed of any missed targets.

The experiment began with a training block of 30 trials. In these trials, the observers tracked 5 out of 10 stimuli for $5 \mathrm{sec}$ without a gap. Following the training block, the observers completed three blocks of experimental trials. Each of these blocks consisted of 10 practice trials and 50 experimental trials, with each trial including a gap. Following the gap, every stimulus reappeared either at the rewind, no-move, or move position (see Figure 2). On rewind trials, the stimuli reappeared at the positions they had occupied 23 frames before the gap began; on no-move trials, they reappeared at the positions they had occupied in the last visible frame before the gap; on move trials, they reappeared at the positions they would have occupied 23 frames after the gap began. Following the gap, each stimulus continued moving for the remainder of the tracking interval. Reappearance position was blocked, so all of the stimuli reappeared at the same relative position throughout each block of trials. The order of conditions was counterbalanced across observers.

\section{Results}

After we removed practice trials, there were 50 trials per observer for each condition. The average proportion of correctly recalled targets is plotted by position in Figure 3 . We submitted the accuracy results to a series of planned comparisons. These revealed that accuracy was reliably lower on move trials (.70) than on either no-move trials $(.74)$ or rewind trials $(.74)[F \mathrm{~s}(1,11)=7.71$ and 8.04 , $M S_{\mathrm{e}} \mathrm{s}=.001, p \mathrm{~s}=.018$ and .016 , respectively]. However, there was no reliable difference between performance on no-move trials and rewind trials $[F(1,11)=0.087$, $\left.M S_{\mathrm{e}}=.001, p=.774\right]$. We performed the same analyses on arcsine-transformed accuracy (Hogg \& Craig, 1995), with qualitatively identical results.

\section{Discussion}

These results replicate those reported by Keane and Pylyshyn (2006). If we compare performance between the no-move and move conditions, the results of Experiment 1 support the location-matching account. Performance was better when the stimuli reappeared at their pregap locations, as would be expected if observers knew only stimulus locations.

However, the observed equivalence between the nomove and rewind conditions is inconsistent with the predictions of the location-matching account. It predicts that performance should decrease with absolute distance between the memorized pregap locations and the reappearance locations (i.e., accuracy should follow an inverted "v" function). These results are also inconsistent with the motion-anticipation account. One explanation is that the observers remembered the paths that targets had traveled just prior to the gap (Keane \& Pylyshyn, 2006). Although this is not consistent with the motion-anticipation account, this explanation does imply some memory for trajectory information.

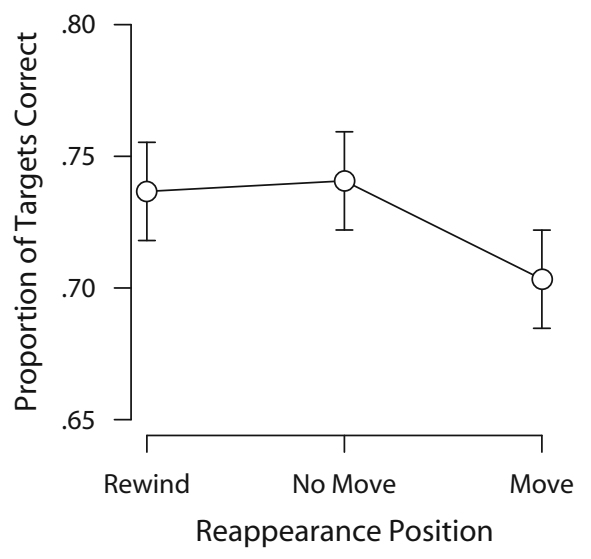

Figure 3. The mean proportion of targets correctly recalled as a function of reappearance position for Experiment 1. Here and in all subsequent figures, error bars indicate $95 \%$ confidence intervals based on within-subjects error (Masson \& Loftus, 2003). 
An alternate explanation is that target reacquisition does not need to occur immediately upon reappearance. Targets that reappear at the rewind position will continue moving and pass through the no-move position $300 \mathrm{msec}$ after the gap. If observers can delay target reacquisition for a few hundred milliseconds on a rewind trial, then they can reacquire targets as if they were performing a no-move trial. Thus, the equivalence between performance on no-move and rewind trials may be due to a confound. We tested this possibility in Experiment 2.

\section{EXPERIMENT 2}

Experiment 2 eliminated the confound present in Experiment 1 , which arose from the fact that each stimulus continued to move forward along its trajectory after the gap, so if it reappeared at the rewind position, it would be at the no-move position $300 \mathrm{msec}$ after it reappeared. To eliminate this confound, we had all of the stimuli stop moving as soon as they reappeared (i.e., the first visible postgap frame was the final frame in the trial). Otherwise, the trials were identical to those in Experiment 1.

In Experiment 2, we also varied the number of targets tracked ("tracking load") by adding blocks in which observers tracked only two targets. ${ }^{2}$ The total number of disks was 10 , regardless of tracking load. Other results from our lab suggest that observers may maintain greater amounts of information for just one or two targets (see Experiments 3-5 and Horowitz et al., 2007). Thus, we might observe support for the motion-anticipation account with two targets, and fail to find such support with five targets.

\section{Method}

The method for Experiment 2 was identical to that of Experiment 1, with the following changes. Three volunteers from Experiment 1, and 9 new volunteers served as observers (ages 19-48, $M=28.7$; 7 females). The disks moved slightly faster, at $9^{\circ} / \mathrm{sec}$. On trials with a gap, all of the disks stopped moving immediately after they reappeared. The total tracking interval varied randomly between $4-6 \mathrm{sec}$ (301-451 frames). Observers tracked either two or five targets. Both reappearance position and tracking load were blocked, so the observers completed six blocks after training. Each block contained 10 practice trials and 40 experimental trials.

\section{Results}

After we removed practice trials, there were 40 trials per observer for each combination of reappearance position and tracking load. The average proportion of correctly recalled targets is plotted by reappearance position and tracking load in Figure 4, along with the results of Experiment 1 . We submitted the proportion correct to a 3 (reappearance position) $\times 2$ (tracking load) repeated measures ANOVA. There was a reliable effect of position on accuracy $\left[F(2,22)=29.5, M S_{\mathrm{e}}=.003, p<.001\right]$. Accuracy was reliably higher when tracking two targets $(.85)$ than when tracking five targets $(.70)\left[F(1,11)=67.9, M S_{\mathrm{e}}=\right.$ $.005, p<.001]$. The interaction between position and tracking load was also reliable, due to the larger effects of reappearance position with two targets than with five targets $\left[F(2,22)=7.80, M S_{\mathrm{e}}=.002, p=.003\right]$. Planned comparisons revealed that, for both two and five targets,

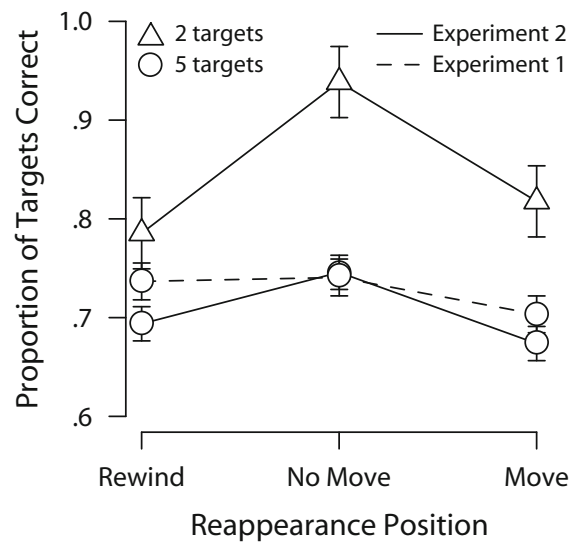

Figure 4. The mean proportion of targets correctly recalled as a function of reappearance position for Experiment 2. Triangles indicate performance with two targets and circles indicate performance with five targets. For comparison, the results for Experiment 1 are plotted as dashed lines.

accuracy was reliably higher on no-move trials (.94 and .75 ) than on either move trials (.82 and .67) or rewind trials (.79 and .69); $p$ s $<.001$. There was no reliable difference in accuracy between move and rewind trials for two or five targets $(F \mathrm{~s}<3, p \mathrm{~s}>.1)$.

We conducted a separate analysis comparing Experiment 1 with Experiment 2. We submitted the proportion correct from both experiments (using only the five-target condition from Experiment 2) to a repeated measures ANOVA with experiment as a between-subjects factor. There was no reliable main effect of experiment $[F(1,22)=$ $\left.0.719, M S_{\mathrm{e}}=.013, p=.406\right]$. However, the interaction between reappearance position and experiment was reliable $\left[F(2,44)=3.93, M S_{\mathrm{e}}=.0009, p=.0270\right]$.

\section{Discussion}

These results indicate that the equivalence in accuracy between no-move and rewind trials observed in Experiment 1 was due to a confound. When the stimuli stopped moving immediately after their reappearance in Experiment 2, observers could use only the postgap locations to reacquire targets; unlike in Experiment 1, they could not wait briefly for the stimuli to move to more effective positions. The results of Experiment 2 show that observers most efficiently reacquired targets when they reappeared in the same positions they occupied just before the gap, and decreased with absolute distance from those positions, consistent with the predictions of the locationmatching account.

Although the effect was still small when observers tracked five targets, it was much larger when they tracked two targets. Unsurprisingly, observers performed better under a lower tracking load. However, reacquisition of targets was more difficult when they had moved away from their pregap positions than when they stayed in the same place, even when observers had to reacquire only two targets. This suggests that the poor performance in the move condition is not due merely to a capacity limit on the amount of motion information that can be retained. 
Rather, it must be the case that either no motion information is available to reacquire targets, or that reacquisition of targets that have not moved since they disappeared is extremely efficient.

\section{EXPERIMENT 3}

Although the results of the previous two experiments support the location-matching account, they do not rule out the motion-anticipation account. In those experiments, it is possible that observers maintained both location and motion information, but they were able to use location information more effectively to find the targets on no-move trials than they could use motion information on move trials. In the remaining experiments, we explicitly test for the use of motion information in target reacquisition. We will demonstrate that a strict location-based account of tracking is insufficient.

We proceed from the observation that, if the locationmatching account is correct and the motion-anticipation account false, then observers in Experiment 2 must have reacquired targets using only the pregap and postgap stimulus locations. That is, they memorized the last visible frame before the gap began and compared that with the locations of the stimuli upon reappearance. Effectively, they memorized a static display, devoid of any motion information. If this is the case, then tracking performance should be unaffected by removing pregap motion information. Experiment 3 tested this possibility.

There were two different conditions in Experiment 3 (see Figure 5): The "motion preview" condition was identical to the move condition used in Experiment 2. In the "static preview" condition, we simply took a motion preview trial and replaced the pregap motion sequence with presentation of the final pregap frame for several seconds. Note that in both conditions, the disks "moved" during the gap. They reappeared at new locations some distance from where they disappeared, and these locations were predictable from a knowledge of their pregap speed and direction. Thus, observers had to make the exact same location comparisons between the pregap and postgap frames in both conditions.

The only difference between the two conditions was in the information available before the gap. In the static preview condition, observers could use only location information to reacquire targets, whereas in the motion preview condition, they could also use the pregap direction and speed of targets to anticipate where they would reappear. The location-matching account predicts that performance in the static preview condition should be equal to (or better than) performance in the motion preview condition. (Performance may be better because pregap locations are presented for several seconds with a static preview, but only briefly with a motion preview.) The motion-anticipation account predicts that performance in the motion preview condition should be better than performance in the static preview condition.

\section{Method}

The method for Experiment 3 was identical to that for Experiment 2, with the following changes. Four volunteers from previous experiments and 8 new volunteers served as observers (ages 19-41, $M=25.9$; 7 females). The total tracking interval, including the $307-$ msec gap, was $4 \sec$ (301 frames) for all of the trials. Following the gap, each disk reappeared as if it had continued moving with constant velocity during the gap, and then stopped immediately (i.e., as in the move condition of Experiment 2).

Trajectories for motion preview trials were generated in the same manner as in previous experiments. Trajectories for static preview

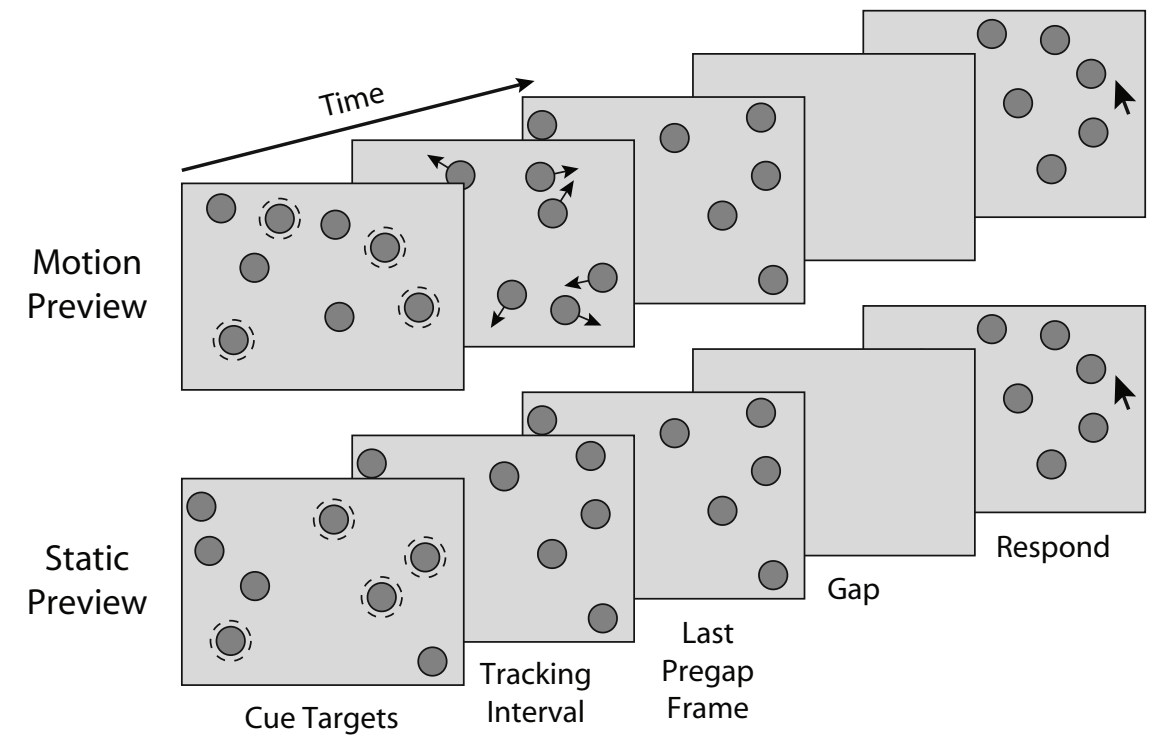

Figure 5. Illustration of the task used in Experiments 3, 4, and 5. Note that all of the frames prior to the gap in the static preview trial are identical to the last pregap frame in the motion preview trial, and that the response frames are identical in both conditions. (See text for more details.) 
trials were generated by taking the last visible pregap frame from a motion preview trial and displaying it statically for $3.7 \mathrm{sec}(277$ frames). From the last pregap frame through the end of the trial (i.e., Frames 278-301), trials in both preview types were identical. The same set of trajectories was used for both preview types.

Observers tracked either one or four targets out of 10 total disks. Both preview type and tracking load were blocked, with the order of blocks counterbalanced across observers. ${ }^{3}$ Each observer completed a total of four blocks, along with an initial training block. Each block contained 10 practice trials and 50 experimental trials.

\section{Results}

After we removed practice trials, there were 50 trials per observer for each combination of preview type and tracking load. The average proportion of correctly recalled targets is plotted by tracking load in Figure 6. We submitted the proportion correct to a 2 (preview type) $\times 2$ (tracking load) repeated measures ANOVA. Accuracy was reliably greater with motion preview (.87) than with static preview $(.83)\left[F(1,11)=8.55, M S_{\mathrm{e}}=.003, p=.0139\right]$. Accuracy was reliably greater with one target (.90) than with four targets $(.81)\left[F(1,11)=48.2, M S_{\mathrm{e}}=.002, p<\right.$ $.001]$. There was also a reliable interaction between preview type and tracking load $\left[F(1,11)=30.6, M S_{\mathrm{e}}=.002\right.$, $p<.001]$. Planned comparisons revealed that accuracy was reliably greater on motion preview trials $(.96)$ than on static preview trials $(.83)$ with one target $(p<.001)$, but marginally lower with four targets (.79 and .82; $p=.091)$. Accuracy declined reliably with tracking load on motion preview trials $(p<.001)$ but not on static preview trials $(p=.552)$.

\section{Discussion}

The results of Experiment 3 reveal a motion preview advantage, showing that observers can make use of motion information to reacquire targets in MOT. Accuracy was higher in the motion preview condition than in the static preview condition with one target, but not with four targets, suggesting that motion information was retained for fewer than four of the targets. Additionally, the fact that the observers were able to recall approximately $80 \%$ of the targets correctly on static preview trials confirms that they can reacquire targets solely on the basis of location information, as was suggested by the results of Experiments 1 and 2 .

\section{EXPERIMENT 4}

In Experiment 3, gap onset always occurred approximately 3,700 msec after the tracking interval began. Thus, it is possible that observers were not utilizing motion information throughout the trial, but only encoded it immediately prior to the gap in order to facilitate target reacquisition. We tested this possibility in Experiment 4 by randomly varying the trial duration between 2 and 5 sec. If observers cannot maintain motion information throughout a trial, then they should perform no better with motion preview than with static preview.

\section{Method}

The method for Experiment 4 was identical to that for Experiment 3, with the following changes. Twelve new volunteers served

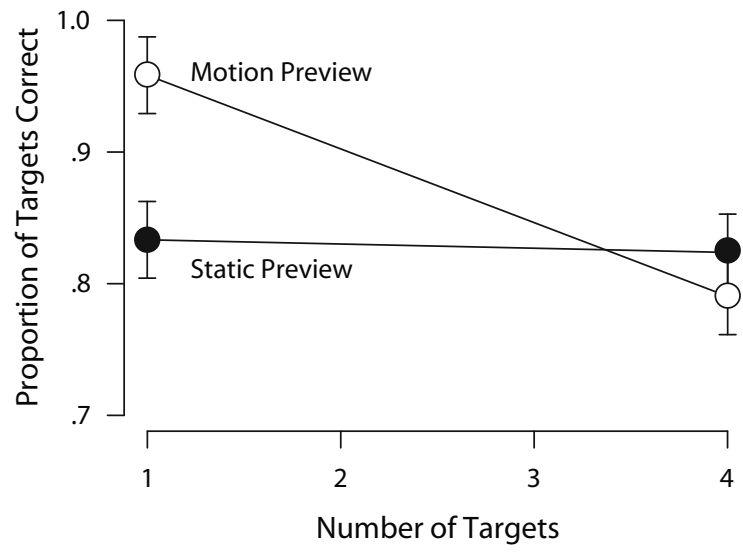

Figure 6. The mean proportion of targets correctly recalled as a function of tracking load for Experiment 3. Open points indicate motion preview trials, and filled points indicate static preview trials.

as observers (records containing age and gender information for these volunteers are missing). The tracking interval was randomly varied in the range of approximately $2-5 \mathrm{sec}$ (151-376 frames). Observers tracked either 2 or 4 out of the 10 disks. Each block contained 10 practice trials and 40 experimental trials.

\section{Results}

After we removed practice trials, there were 40 trials per observer for each combination of preview type and tracking load. The average proportion of correctly recalled targets is plotted by tracking load in Figure 7. We submitted the proportion correct to a 2 (preview type) $\times 2$ (tracking load) repeated measures ANOVA. There was no reliable difference in accuracy between motion preview trials $(.81)$ and static preview trials $(.80)[F(1,11)=1.21$, $\left.M S_{\mathrm{e}}=.004, p=.29\right]$. Accuracy was reliably greater with two targets $(.84)$ than with four targets $(.77)[F(1,11)=$ $\left.34.50, M S_{\mathrm{e}}=.002, p<.001\right]$. There was also a reliable interaction between preview type and tracking load

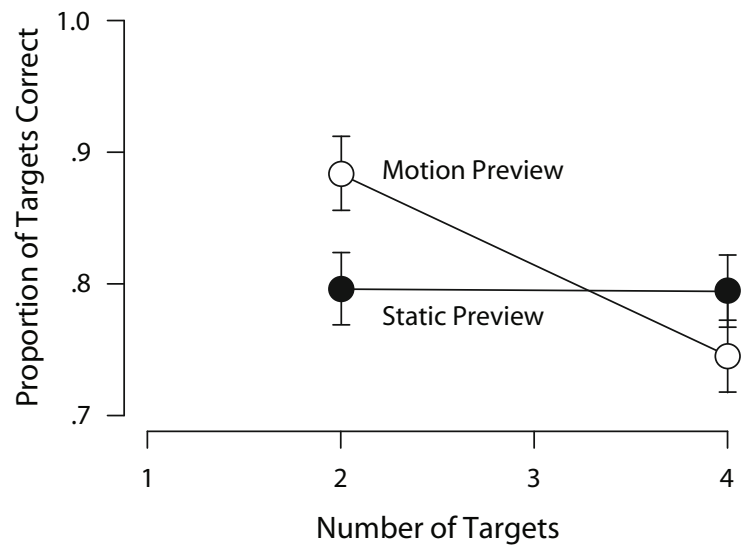

Figure 7. The mean proportion of targets correctly recalled as a function of tracking load for Experiment 4. Open points indicate motion preview trials, and filled points indicate static preview trials. 
$\left[F(1,11)=43.76, M S_{\mathrm{e}}=.001, p<.001\right]$. Planned comparisons revealed that accuracy was reliably greater with motion preview (.88) than with static preview (.80) with two targets $(p<.001)$, but reliably lower with four targets $(.74$ and $.79 ; p=.04)$. Accuracy declined reliably with tracking load on motion preview trials $(p<.001)$ but not on static preview trials $(p=.93)$.

\section{Discussion}

The results of Experiment 4 are consistent with those of Experiment 3: The observers performed better with a motion preview than with a static preview when tracking two targets, but not when tracking four targets. Since the observers could not have predicted gap onset in Experiment 4 , they must have been able to maintain motion information throughout the trial. Once again, the interaction between preview type and tracking load suggests that the observers maintained motion information for one to three stimuli.

\section{EXPERIMENT 5}

The results of Experiment 3 and Experiment 4 supported the hypothesis that observers can retain motion information for a limited number of moving objects. However, those results did not allow us to measure the actual number of objects for which motion information was retained. We designed Experiment 5 in order to quantify the number of objects' worth of information that must be retained in order to obtain the observed levels of performance. To accomplish this, we systematically varied tracking load from one to four targets in both preview conditions, and developed a model to specify the relationship between the number of targets tracked and accuracy in the task.

The primary purpose of the model is to estimate the number of objects for which observers must have maintained motion information in order to obtain the observed motion preview advantage. It assumes that observers can track moving stimuli with one of two mechanisms: One mechanism stores only location information and the other extrapolates motion information. During the gap, information from both mechanisms is stored, and may be used to reacquire target objects.

Motion information may be used to anticipate postgap reappearance positions with high levels of accuracy, since all stimuli continue moving along their trajectories during the gap. Although location information may also be used to reacquire targets, it is less accurate, since the postgap locations are different from the memorized locations.

The probability of successfully reacquiring a target following the gap on motion preview trials depends on both motion and location information. However, there is no motion information available prior to the gap on static preview trials, so reacquisition depends only on location information.

There are four possible free parameters in the model. The capacity of the location-based mechanism is $k_{1}$, and the capacity of the motion-based mechanism is $k_{\mathrm{m}}$. There are two probabilities of reacquiring targets on the basis of location information, one for each preview type: $p_{\mathrm{s}}$ for static preview trials and $p_{\mathrm{m}}$ for motion preview trials. We used different probabilities for each preview type because locations were available for longer on static preview trials than on motion preview trials, so it seemed reasonable to assume that $p_{\mathrm{s}} \neq p_{\mathrm{m}}$.

We can reduce the number of parameters by assuming that $k_{1} \geq 4$ (i.e., location capacity is at least four objects). There were never more than four targets in Experiment 5, so this assumption means that location information was retained for all targets, causing the location-based mechanism to have effectively no capacity limit. This assumption is based on several facts. First, we are primarily interested in precise estimates of capacity for motion information, and estimates of location-based capacity are only useful as a baseline. Second, previous MOT studies have found tracking capacity to be around four to five objects (e.g., Pylyshyn \& Storm, 1988). Finally, tracking load had no effect on performance in static preview trials in Experiments 3 and 4 (nor in Experiment 5, to anticipate our results). Had the location-based mechanism been over capacity, performance would have declined with tracking load. Instead, it appears that location information was stored for at least four targets.

The model makes quantitative predictions about the relationship between the three remaining parameters $\left(k_{\mathrm{m}}\right.$, $p_{\mathrm{m}}$, and $p_{\mathrm{s}}$ ), the number of targets, and tracking performance. If there are $t$ targets, then on motion preview trials, the predicted number of correctly recalled targets, $n$, is

$$
n=k_{\mathrm{m}}+p_{\mathrm{m}}\left(t-k_{\mathrm{m}}\right) \text {, }
$$

unless $k_{\mathrm{m}}>t$, in which case $n=t$. On static preview trials,

$$
n=p_{\mathrm{s}} t
$$

The proportion of correctly recalled targets can be calculated by dividing Equations 1 and 2 by $t$.

\section{Method}

The method for Experiment 5 was identical to that for Experiment 3, with two changes. First, 5 volunteers from previous experiments and 7 new volunteers served as observers (ages 20-37, $M=$ 27.3; 6 females). Second, observers tracked 1, 2, 3, or 4 out of 10 disks. The two factors (tracking load and preview type) were manipulated between block, for a total of eight blocks (plus one initial training block), each with 10 practice trials and 50 experimental trials.

\section{Results}

After we removed practice trials, there were 50 trials per observer for each combination of preview type and tracking load. The average proportion of correctly recalled targets is plotted by tracking load in Figure 8 (solid lines). We submitted the proportion correct to a 2 (preview type) $\times 4$ (tracking load) repeated measures ANOVA. Accuracy was reliably greater on motion preview trials (.88) than on static preview trials $(.81)\left[F(1,11)=58.69, M S_{\mathrm{e}}=\right.$ $.002, p<.001]$. The main effect of tracking load was reliable $\left[F(3,33)=16.51, M S_{\mathrm{e}}=.002, p<.001\right]$. There was also a reliable interaction between preview type and tracking load $\left[F(3,33)=19.46, M S_{\mathrm{e}}=.002, p<.001\right]$. 


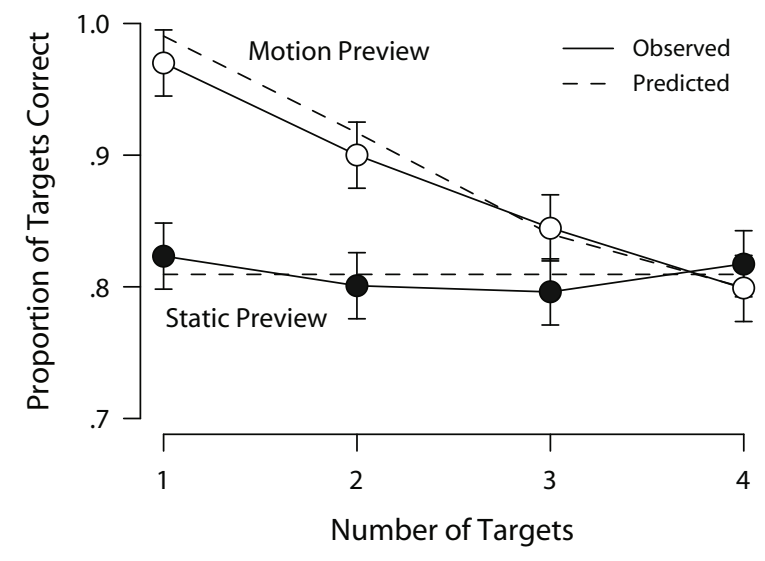

Figure 8. The mean observed and predicted proportion of targets correctly recalled as a function of tracking load for Experiment 5. Open points indicate motion preview trials, and filled points indicate static preview trials. Solid lines indicate observed performance and dashed lines indicate performance predicted by the fitted model.

Planned comparisons revealed that accuracy was reliably greater in the moving condition than in the static condition with one (.97 and .82), two (.90 and .80), and three (.84 and .80$)$ targets $(p \mathrm{~s}<.01)$, but not reliably different with four targets $(.80$ and $.82 ; p=.289)$. We fit a linear model to the proportion of correctly recalled targets across tracking load for each preview type separately. The slope was reliable for the motion preview condition [slope $=-.057$; $t(2)=-14.93, S E=.004, p=.004]$ but not for the static preview condition [slope $=-.0022 ; t(2)=-0.32, S E=$ $.007, p=.780]$.

We estimated the model's parameters $\left(k_{\mathrm{m}}, p_{\mathrm{m}}, p_{\mathrm{s}}\right)$ using a gradient descent algorithm. This allowed us to find the parameter values that minimized the sum-squared error between the observed and predicted data for each observer. Each observer contributed eight data points, leaving $5 d f$ s per observer. The average estimated parameter values were $k_{\mathrm{m}}=1.47 \pm 0.29, p_{\mathrm{m}}=.68 \pm .06$, and $p_{\mathrm{s}}=$ $.81 \pm .04$ (the ranges are $95 \%$ confidence intervals). The average predicted proportion of correctly recalled targets is indicated by the dashed lines in Figure 8. For the averaged data, $R^{2}=.977$.

\section{Discussion}

The results reveal a systematic decline in performance with tracking load on motion preview trial blocks, but no change in performance on static preview trial blocks. The average estimated capacity for motion information was approximately 1.5 objects. On average, the observers stored more than 1 object, meaning that observers must have the capacity to store at least 2 objects' worth of motion information. Thus, the motion preview advantage indicates that observers are able to keep track of the motion of 2 targets in addition to whatever location information they track (which seems to be tracked for at least 4 objects). The results of Experiment 5 clearly support the motionanticipation account of target reacquisition.

\section{GENERAL DISCUSSION}

These experiments reveal two basic facts. First, the observers reacquired targets most efficiently after a brief disappearance when the targets reappeared in the same positions they occupied before disappearing (Experiments 1 and 2). This replicates Keane and Pylyshyn (2006), and is consistent with their interpretation that observers maintain only location information for tracked targets. Second, the observers' performance was better with pregap motion information than without, clearly demonstrating that they could make use of motion information to track moving objects, and reacquire them after they reappeared (Experiment 3, 4, and 5).

The apparent contradiction between these two findings can be readily resolved. The superiority of the no-move condition over the move and rewind conditions in Experiment 2 indicates that location information is retained during disappearance and presents the most effective means for matching targets across the gap. However, it does not rule out storage of motion information. The gap introduces uncertainty into the MOT task because observers cannot maintain object continuity using visible stimuli. This uncertainty is minimized in the no-move condition. Motion information is also remembered for some stimuli and can be used to reduce uncertainty when stimuli continue moving during the gap, leading to better performance with motion preview than with static preview.

Clearly, the visual system retains both location and motion information for tracked objects. The results suggest that observers can track location information for at least four targets and motion information for about two targets. The fact that estimated capacity is greater than one object is inconsistent with the possibility that eye movements following a single target might subserve anticipation of reappearance position (Kerzel, Jordan, \& Müsseler, 2001). Covert shifts of attention split among multiple targets (Awh \& Pashler, 2000) might serve this purpose instead (Oksama \& Hyönä, 2004).

There must be representations that can be used to track location and motion information. Visual indexes alone cannot fulfill this requirement, since indexes do not extract or encode any information beyond an object's spatial location (Pylyshyn, 1989, 2004). However, two independent representations could readily account for our results. Our recent work on tracking unique, identifiable objects (Horowitz et al., 2007) suggests that there may be independent representations for the locations and identities of tracked targets (see also Oksama \& Hyönä, 2004). Observers' capacity for identity information in those studies was similar to the capacity for motion information in the present experiments. Horowitz et al. (2007) tentatively suggested that location information might be served by visual indexes, whereas identity information required object file representations (Kahneman et al., 1992).

Our present results are compatible with this proposal. For example, while tracking objects in Experiment 2, observers could have used visual indexes to maintain the current locations of about four targets. Additionally, they 
could have extracted motion information (e.g., past trajectory, current direction of motion) from two of the four targets and stored it in object files. Assuming the information stored in the visual indexes and object files could be retained during the gap, then this information could be used to reacquire the targets following the gap.

If the objects reappeared in their pregap positions, then the location information stored in the visual indexes could be used to match remembered positions with actual positions. The precision of this reacquisition depends on the fidelity of the location information stored by the visual indexes. If the objects moved during the gap - either forward or backward along their pregap trajectories - then motion information could be used to calculate the anticipated reappearance positions of the two targets for which object files were retained. The precision of such reacquisition depends on the fidelity of the motion information stored by object files. Any remaining targets could be reacquired by searching around their memorized pregap locations. The accuracy of this search depends on the proximity of other objects to the memorized locations.

How do we know whether observers in our experiments actually tracked location and motion information continuously during the gap? The results of Scholl and Pylyshyn (1999) and Scholl and Feigenson (2004) suggest that tracking stops for objects that disappear spontaneously. However, we have proposed that observers treat simultaneous disappearance as a brief interruption of the task, and use a task-postponement system to store information relevant to the tracking task offline during the gap (Alvarez et al., 2005; Horowitz et al., 2006). This means that the objects are not being actively tracked during the gap. However, in order for information to be stored during the gap, it must have been available prior to the gap. Since gap onset is unpredictable, any information stored during the gap must be continuously available during the tracking interval. Therefore, any motion information stored during the gap must be continuously tracked during visible motion.

According to our results, then, a driver tracks both the location of the cars around her and their speed and direction of motion. Although she can track motion information for only one or two cars, that should be sufficient to track the nearest cars and anticipate potentially dangerous situations. Similarly, a parent watching his children and their friends on a playground may be able to track how two of them are running while knowing the current locations of some of the others. If he looks away momentarily, he should be able to anticipate where two of them will be when he looks back (except when they turn), and will have to search around for the remaining children on the basis of where they were before.

The technique introduced in Experiment 3 allows us to distinguish memory for motion information from memory for location information. This opens up avenues for future research on the characteristics of these two types of information. One question worth investigating is: How much motion does the visual system need in order to create a useful representation? Can an object's speed and direction be determined on the basis of two frames, or does it take hundreds of milliseconds? Another question is whether distraction can have differential effects on the two types of information. Alvarez et al. (2005) showed that a visual search task presented during a gap in MOT had only a minimal effect on tracking performance. This finding could be specific to location information, and it may be that a distracting task does interfere with storage of motion information. Such a dissociation would support the hypothesis that location and motion information are stored by separate representations.

\section{AUTHOR NOTE}

Portions of this article were presented at a meeting of the Vision Sciences Society (Fencsik, Horowitz, Klieger, \& Wolfe, 2004). This research was supported by NIMH Grant R01-65576 to T.S.H. We thank Lana Trick for comments on the manuscript, and George Alvarez, Brian Keane, and Jeremy Wolfe for their valuable insights and suggestions. Correspondence concerning this article should be addressed to D. E. Fencsik, who is now at the Psychology Department, California State University East Bay, Hayward, CA 94542 (e-mail: david.fencsik@csueastbay.edu) or to T. S. Horowitz, Visual Attention Laboratory, Brigham and Women's Hospital, 64 Sidney Street, Suite 170, Cambridge, MA 02139 (toddh@) search.bwh.harvard. edu).

\section{REFERENCES}

Alvarez, G. A., Horowitz, T. S., Arsenio, H. C., DiMase, J. S., \& Wolfe, J. M. (2005). Do multielement visual tracking and visual search draw continuously on the same visual attention resources? Journal of Experimental Psychology: Human Perception \& Performance, 31, 643-667.

Awh, E., \& PAshler, H. (2000). Evidence for split attentional foci. Journal of Experimental Psychology: Human Perception \& Performance, 26, 834-846.

BAHRAMI, B. (2003). Object property encoding and change blindness in multiple object tracking. Visual Cognition, 10, 949-963.

Brainard, D. H. (1997). The Psychophysics Toolbox. Spatial Vision, 10, 433-436.

Fencsik, D. E., Horowitz, T. S., Klieger, S. B., \& Wolfe, J. M. (2004, May). Target reacquisition strategies in multiple object tracking. Poster session presented at the annual meeting of the Vision Sciences Society, Sarasota, FL.

Finke, R. A., Freyd, J. J., \& Shyi, G. C.-W. (1986). Implied velocity and acceleration induce transformations of visual memory. Journal of Experimental Psychology: General, 115, 175-188.

HogG, R. V., \& CRAIG, A. T. (1995). Introduction to mathematical statistics (5th ed.). Upper Saddle River, NJ: Prentice Hall.

Horowitz, T. S., Birnkrant, R. S., Fencsik, D. E., Tran, L., \& Wolfe, J. M. (2006). How do we track invisible objects? Psychonomic Bulletin \& Review, 13, 516-523.

Horowitz, T. S., Klieger, S. B., Fencsik, D. E., Yang, K. K., Alvarez, G., \& Wolfe, J. M. (2007). Tracking unique objects. Perception \& Psychophysics, 69, 172-184.

Kahneman, D., Treisman, A., \& Gibbs, B. J. (1992). The reviewing of object files: Object-specific integration of information. Cognitive Psychology, 24, 175-219.

Keane, B. P., \& Pylyshyn, Z. W. (2006). Is motion extrapolation employed in multiple object tracking? Tracking as a low-level, nonpredictive function. Cognitive Psychology, 52, 346-368.

Kerzel, D., Jordan, J. S., \& Müsseler, J. (2001). The role of perception in the mislocalization of the final position of a moving target. Journal of Experimental Psychology: Human Perception \& Performance, 27, 829-840.

Klieger, S. B., Horowitz, T. S., \& Wolfe, J. M. (2004). Is multiple object tracking colorblind? [Abstract]. Journal of Vision, 4, 363a, journalofvision.org/4/8/363.

Masson, M. E. J., \& LofTus, G. R. (2003). Using confidence intervals for graphically based data interpretation. Canadian Journal of Experimental Psychology, 57, 203-220.

NisSEN, M. J. (1985). Accessing features and objects: Is location special? 
In M. I. Posner \& O. S. M. Marin (Eds.), Attention and performance $X I$ (pp. 205-219). Hillsdale, NJ: Erlbaum.

OKSAMA, L., \& HYÖNÄ, J. (2004). Is multiple object tracking carried out automatically by an early vision mechanism independent of higherorder cognition? An individual difference approach. Visual Cognition, 11, 631-671.

Pelli, D. G. (1997). The VideoToolbox software for visual psychophysics: Transforming numbers into movies. Spatial Vision, 10, 437-442.

PyLyshyn, Z. W. (1989). The role of location indexes in spatial perception: A sketch of the FINST spatial-index model. Cognition, 32, 65-97.

Pylyshyn, Z. W. (2001). Visual indexes, preconceptual objects, and situated vision. Cognition, 80, 127-158.

Pylyshyn, Z. W. (2004). Some puzzling findings in multiple object tracking: I. Tracking without keeping track of object identities. Visual Cognition, 11, 801-822.

Pylyshyn, Z. W., \& Storm, R. W. (1988). Tracking multiple independent targets: Evidence for a parallel tracking mechanism. Spatial Vision, 3, 1-19.

Scholl, B. J., \& Feigenson, L. (2004). When out of sight is out of mind: Perceiving object persistence through occlusion vs. implosion [Abstract]. Journal of Vision, 4, 26a, journalofvision.org/4/8/26.

Scholl, B. J., \& Pylyshyn, Z. W. (1999). Tracking multiple items through occlusion: Clues to visual objecthood. Cognitive Psychology, 38, 259-290.

Scholl, B. J., Pylyshyn, Z. W., \& Franconeri, S. L. (1999, May) When are spatiotemporal and featural properties encoded as a result of attentional allocation? Paper presented at the annual meeting of the Association for Research in Vision and Ophthalmology, Ft. Lauderdale, FL. [Abstract published in Investigative Ophthalmology \& Visual Science, 40, S797].

Vogel, E. K., Woodman, G. F., \& Luck, S. J. (2001). Storage of features, conjunctions, and objects in visual working memory. Journal of Experimental Psychology: Human Perception \& Performance, 27, 92-114.

Wolfe, J. M. (1998). Visual search. In H. Pashler (Ed.), Attention (pp. 13-73). Philadelphia: Taylor \& Francis.

YanTIS, S. (1992). Multielement visual tracking: Attention and perceptual organization. Cognitive Psychology, 24, 295-340.

\section{NOTES}

1. No age information was recorded for 2 of the participants, 1 female and 1 male.

2. Tracking load was varied between blocks in order to simplify the task for observers. Mixing tracking load within blocks cannot have any substantive effect on tracking performance, because observers know the tracking load throughout the tracking interval.

3. There is no need to randomize either preview type or tracking load, since both will be readily apparent to an observer from the beginning of the trial.

(Manuscript received March 31, 2005; revision accepted for publication September 26, 2006.) 